Revista Española de Cirugía Ortopédica y Traumatología, vol. 60, núm. 1, 2015, pp. 29-37.

\title{
Analysis of judicial sentences issued against traumatologists between 1995 and 2011 as regards medical negligence.
}

Cardoso Cita, Zulema, Perea Pérez, Bernardo, Albarrán Juan, María Elena, Labajo González, Elena, López Durán, Luis, Marco Martínez, Fernando y Santiago Sáez, Andrés.

Cita:

Cardoso Cita, Zulema, Perea Pérez, Bernardo, Albarrán Juan, María Elena, Labajo González, Elena, López Durán, Luis, Marco Martínez, Fernando y Santiago Sáez, Andrés (2015). Analysis of judicial sentences issued against traumatologists between 1995 and 2011 as regards medical negligence. Revista Española de Cirugía Ortopédica y Traumatología, 60 (1), 29-37.

Dirección estable: https://www.aacademica.org/elenalabajogonzalez/12 ARK: https://n2t.net/ark:/13683/pcQr/yaN

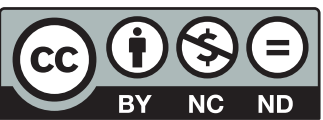




\title{
Revista Española de Cirugía Ortopédica y Traumatología
}

www.elsevier.es/rot

ORIGINAL

\section{Análisis de sentencias judiciales relativas a negligencias médicas emitidas contra traumatólogos entre 1995 y 2011}

\author{
Z. Cardoso-Citaa ${ }^{\mathrm{a}, *}$, B. Perea-Pérez ${ }^{\mathrm{b}}$, M.E. Albarrán-Juan ${ }^{\mathrm{b}}$, M.E. Labajo-González ${ }^{\mathrm{b}}$, \\ L. López-Durán ${ }^{a}$, F. Marco-Martínez ${ }^{\mathrm{a}}$ y A. Santiago-Saéz ${ }^{\mathrm{b}, \mathrm{c}}$
}

a Servicio de Traumatología y Cirugía Ortopédica, Hospital Clínico San Carlos, Madrid, España

${ }^{\mathrm{b}}$ Escuela de Medicina Legal, Universidad Complutense de Madrid, Madrid, España

c Servicio de Medicina Legal, Hospital Clínico San Carlos, Madrid, España

Recibido el 15 de septiembre de 2014; aceptado el 18 de julio de 2015

Disponible en Internet el 5 de septiembre de 2015

\author{
PALABRAS CLAVE \\ Mala praxis; \\ Negligencia médica; \\ Condena; \\ Consentimiento \\ informado; \\ Traumatología y \\ Cirugía Ortopédica
}

\begin{abstract}
Resumen
Introducción y objetivos: La Traumatología y Cirugía Ortopédica es una de las especialidades más reclamadas por su amplitud y complejidad. Nuestro objetivo es determinar las características de las reclamaciones presentadas contra médicos especialistas en Traumatología, considerando todas aquellas variables que puedan tener influencia tanto en el planteamiento de la demanda como en la resolución del proceso.

Material y métodos: Se ha realizado un análisis de 303 sentencias judiciales (1995-2011) recogidas en el archivo de sentencias judiciales sanitarias de la Escuela de Medicina Legal de Madrid que se nutre de la base de datos de Westlaw Aranzazi.

Resultados: La jurisdicción civil fue la más empleada. El proceso específico más reclamado fueron los trastornos osteoarticulares seguidos de las alteraciones vasculonerviosas y de las infecciones. La lesión reclamada ocurrió con más frecuencia en miembros inferiores sobre todo rodilla. La causa general de reclamación más frecuente fue el error terapéutico quirúrgico seguido del error diagnóstico. En el 14,9\% fue el defecto de información. Existió condena en el 49,8\% de los casos siendo la indemnización mayoritariamente menor de 50.000 euros.

Conclusiones: Concluimos que la Traumatología y Cirugía Ortopédica es una especialidad proclive a las reclamaciones por mala praxis. El número de condenas a traumatólogos es elevado pero las indemnizaciones suelen ser menores de 50.000 euros. El motivo fundamental de las reclamaciones es el error terapéutico quirúrgico siendo pues el acto quirúrgico fundamental y donde se deben extremar las precauciones. Las condenas por deficiente información son elevadas siendo fundamental una adecuada comunicación médico-paciente y rellenar correctamente el consentimiento informado.

(c) 2014 SECOT. Publicado por Elsevier España, S.L.U. Todos los derechos reservados.
\end{abstract}

\footnotetext{
* Autor para correspondencia.

Correo electrónico: zulemacardoso@hotmail.com (Z. Cardoso-Cita).
} 


\section{KEYWORDS}

Malpractice;

Medical malpractice;

Sentences;

Informed consent;

Traumatology and

Orthopaedic Surgery
Analysis of judicial sentences issued against traumatologists between 1995 and 2011 as regards medical negligence

\begin{abstract}
Introduction and objectives: Traumatology and Orthopaedic Surgery is one of the specialities with most complaints due to its scope and complexity. The aim of this study is to determine the characteristics of the complaints made against medical specialists in Traumatology, taking into account those variables that might have an influence both on the presenting of the complaint as well as on the resolving of the process.

Material and methods: An analysis was performed on 303 legal judgments (1995-2011) collected in the health legal judgements archive of the Madrid School of Medicine, which is linked to the Westlaw Aranzadi data base.

Results: Civil jurisdiction was the most used. The specific processes with most complaints were bone-joint disorders followed by vascular-nerve problems and infections. The injury claimed against most was in the lower limb, particularly the knee. The most frequent general cause of complaint was surgical treatment error, followed by diagnostic error. There was lack of information in $14.9 \%$. There was sentencing in $49.8 \%$ of the cases, with compensation mainly being less than 50,000 euros.

Conclusions: Traumatology and Orthopaedic Surgery is a speciality prone to complaints due to malpractice. The number of sentences against traumatologists is high, but compensations are usually less than 50,000 euros. The main reason for sentencing is surgical treatment error; thus being the basic surgical procedure and where precautions should be maximised. The judgements due to lack of information are high, with adequate doctor-patient communication being essential as well as the correct completion of the informed consent.

๑ 2014 SECOT. Published by Elsevier España, S.L.U. All rights reserved.
\end{abstract}

\section{Introducción}

Siendo la Traumatología y Cirugía Ortopédica una de las especialidades más reclamadas debido a su amplitud y complejidad, en este artículo pretendemos determinar las características de las reclamaciones presentadas contra médicos especialistas en Traumatología y Cirugía Ortopédica, considerando todas aquellas variables que puedan tener influencia tanto en el planteamiento de la demanda como en la resolución del proceso.

El incremento de la judicialización de los asuntos sanitarios en España afecta a todas las especialidades ${ }^{1}$, sean quirúrgicas o médicas, hospitalarias o extrahospitalarias. Durante el periodo de 1995 a 1998 se presentaron contra el sistema nacional de salud 932 reclamaciones (judiciales y extrajudiciales).

Los errores médicos costaron al extinto INSALUD 726,5 millones de las antiguas pesetas por indemnizaciones a 95 pacientes (una media de 7,8 millones de pesetas por paciente $)^{2}$.

Por especialidades, Medicina de Urgencia supuso el 23,8\% de las reclamaciones, Ginecología y Obstetricia el $18,2 \%$, Traumatología y Cirugía Ortopédica el $11,2 \%$ y Neurocirugía el $4,6 \%^{2}$. Estos datos son concordantes con el estudio realizado por Torre de la compañía de seguros Willis con datos del reaseguro de los servicios públicos de salud en España durante el periodo 2005-2007 ${ }^{3}$. Un estudio del Nacional Health Service Británico informó que se cerraron 3.254 reclamaciones durante el periodo 1999-2000, con un coste de 386 millones de libras esterlinas ${ }^{4}$.
Una consecuencia inmediata de esta situación puede ser la práctica sistemática de la medicina defensiva ${ }^{5,6}$, que se aleja de la relación fluida entre médico y paciente, basada en la confianza mutua y con una orientación bioética que potencia la propia autonomía del paciente.

Sin embargo, un análisis de los factores que motivan una reclamación y de los condicionamientos que influyen en las decisiones judiciales pueden ayudar a los especialistas en traumatología y cirugía ortopédica a valorar determinadas prácticas clínicas de forma especial, extremando las precauciones ante aquellos signos que puedan dar lugar a una reclamación judicial.

En la práctica de la traumatología y cirugía ortopédica existen particularidades ${ }^{7}$ que conviene señalar y que hacen que esta especialidad sea muy proclive a las reclamaciones por responsabilidad profesional ${ }^{8-10}$ :

- Pacientes con características especiales tales como los politraumatizados en los que la comunicación médico paciente es inexistente y la atención que precisan es multidisciplinar participando variadas especialidades médicas y quirúrgicas incrementándose el riesgo de reclamaciones judiciales.

- Fracturas tratadas de urgencia cuyo tratamiento se realiza en condiciones difíciles y adversas.

- Tratamiento de adultos jóvenes que esperan mayores expectativas del resultado tras el tratamiento.

- Empleo de implantes (prótesis y material de osteosíntesis) ${ }^{11}$. 
- Necesaria colaboración activa del paciente en el tratamiento (rehabilitación...)

- Existencia de numerosas subespecialidades en la Traumatología y Cirugía Ortopédica con gran número de procedimientos específicos en cada una de ellas (mano, columna, pie...).

En el momento actual la mayor parte de la literatura médica publicada sobre negligencias médicas son relativas al ámbito estadounidense, pero el análisis del escenario en este país es diferente al que podemos encontrar en nuestro medio ya que existen notables diferencias entre el sistema sanitario y jurídico español y el de los Estados Unidos.

Arymani-Manso et al. ${ }^{12}$ nos realizan una aproximación mucho más cercana a nuestro entorno, reflejando datos de aseguradoras en Cataluña donde el 1,33\% de los médicos asegurados se enfrentaron a alguna reclamación si bien solo el $0,26 \%$ tuvo que abonar una indemnización al reclamante.

En nuestro ámbito nacional disponemos de estudios que nos aportan datos fundamentales tales como los factores de riesgo implicados en cirugías de sitio erróneo, entre los que destacan las intervenciones de urgencia, cirugías múltiples y simultáneas en un mismo paciente, obesidad o deformidades en los pacientes, falta de familiaridad con el instrumental que se va a usar etc. ${ }^{13}$.

\section{Objetivos}

El objetivo del presente estudio es analizar las sentencias judiciales emitidas en segunda instancia presentadas contra especialistas en traumatología y cirugía ortopédica, estableciendo la tipología del procedimiento médico o quirúrgico reclamado y analizando la resolución de las sentencias, considerando si existe condena y su cuantía económica.

De este modo los resultados podrán facilitar una práctica más segura de la especialidad, tanto para el paciente como para el médico.

No existe en la literatura de los últimos años ningún estudio similar que haga referencia específica a la especialidad de Traumatología y Cirugía Ortopédica en España.

\section{Material y métodos}

El presente estudio se ha realizado sobre el «archivo de sentencias judiciales sanitarias de la Escuela de Medicina Legal de Madrid», de la Universidad Complutense de Madrid. Este archivo contiene 2.817 sentencias judiciales de segunda o última instancia referidas a actuaciones sanitarias emitidas por las jurisdicciones civil, penal y contencioso administrativa durante el periodo comprendido entre 1995 y enero de 2011.

El citado archivo se nutre de las sentencias publicadas por la base de datos de CENDOJ y Westlaw Aranzazi que durante el periodo de estudio recogía la totalidad de sentencias judiciales de segunda instancia o posterior publicadas.

Las sentencias de primera instancia no se publican uniformemente en ninguna base de datos.

Tenemos que aclarar que la jerarquía jurisdiccional española se establece por su función procesal, podemos distinguir por un lado, los órganos de primera o única instancia que son aquellos que resuelven los asuntos por primera vez, pero en determinados casos (cuando existe un desacuerdo en la sentencia) cabe una segunda instancia, es decir un nuevo enjuiciamiento ante un órgano superior (Tribunal Superior de Justicia, Tribunal Supremo y Audiencia Nacional).

Para realizar el presente estudio se han seleccionado de la totalidad de las 2.817 sentencias relativas a mala praxis médica recogidas en el archivo las 303 que cumplían los siguientes criterios de inclusión:

1. Sentencia referida a un médico especialista en traumatología y cirugía ortopédica, debido al ejercicio de su especialidad, según definición y maniobras contempladas en la ORDEN SCO/226/2007, de 24 de enero, por la que se aprueba y publica el programa formativo de la especialidad de Cirugía Ortopédica y Traumatología en España.

2. Se han excluido expresamente las sentencias que si bien el acto quirúrgico o el tratamiento realizado eran propios de la especialidad de Traumatología y Cirugía Ortopédica, en ese caso concreto fue realizado por un profesional distinto al traumatólogo (podólogo, fisioterapeuta etc...).

3. Texto de la sentencia en la que figure el motivo de la reclamación, las circunstancias y el lugar en el que se produjo, sus consecuencias y la resolución judicial.

Se ha realizado una ficha de recogida de datos relativa a cada sentencia judicial donde se han implementado diferentes variables que serán detalladas en el apartado de resultados.

Tras realizar un estudio descriptivo de las variables, se ha realizado un contraste de variables mediante tablas de contingencia mediante la prueba de Chi cuadrado utilizando el programa estadístico SPSS 15 estableciéndose un nivel de significación estadística del 95\% $(\mathrm{p}<0,05)$.

Para una mejor interpretación de las tablas que contienen los resultados, indicamos que existen dos categorías analizadas en el presente trabajo y que están íntimamente relacionadas e incluso pueden confundirse entre sí y son las causas específicas y las causas generales que motivaron la reclamación, por ello realizamos las siguientes indicaciones:

- Las causas específicas nos indican de manera concreta la lesión que motivó directamente la reclamación:

- Infección: a lo largo de un tratamiento ortopédico o quirúrgico se produjo una infección que fue la que motivó la reclamación.

- Lesión vascular o nerviosa.

- En el curso de su tratamiento el paciente desarrolló un síndrome compartimental.

- En su evolución el paciente presentó algún tipo de complicación añadida (sangrado, tromboembolismo, trombosis venosa, patología cardiaca, patología pulmonar...) que hemos decidido denominarlas en conjunto como complicaciones sistémicas.

- En la categoría de alteraciones osteoarticulares hemos incluido los dolores articulares residuales, pérdida de movilidad articular, deformidades articulares...

- En la categoría de causas generales, aunque íntimamente relacionada con las causas específicas, hemos querido diseñar una categoría más amplia de causas de reclamación: 
- Defecto de información: que incluye tanto la falta de consentimiento informado como defectos en su realización.

- Error diagnóstico: el paciente alega en su reclamación un error al ser diagnosticado incluyéndose en esta categoría fracturas que han pasado desapercibidas en una primera evaluación.

- Error terapéutico ortopédico: el paciente indica como motivo de reclamación un error en su tratamiento no quirúrgico (mala colocación de yesos etc....).

- Error terapéutico quirúrgico: el paciente indica como causa de reclamación un error en el transcurso de una intervención quirúrgica.

- Lado equivocado: el reclamante es intervenido del miembro contralateral.

- En la categoría de mal seguimiento médico se incluyen causas de reclamación en el transcurso del tratamiento quirúrgico u ortopédico tales como retraso diagnóstico de infecciones, errores en la prescripción farmacológica, no prescripción de fármacos antitrombóticos etc.).

\section{Resultados (tablas 1-3)}

Una vez estudiadas las 303 sentencias emitidas en segunda instancia relativas a negligencias en traumatología obtenemos los siguientes resultados:.

La jurisdicción civil ha sido la más frecuentemente empleada para plantear las reclamaciones judiciales contra traumatólogos y cirujanos ortopédicos $(88,4 \%)$, siendo las audiencias provinciales las que mayor número de sentencias han dictado $(75,9 \%)$.

La Comunidad Autónoma con mayor número de sentencias dictadas es Cataluña $(28,7 \%)$ seguida de Madrid y Andalucía (11,6\% en cada una de ellas).

El ámbito asistencial más frecuentemente implicado fue la medicina privada $(63,75 \%)$.

En el sexo de los pacientes implicados la proporción registrada fue similar (46,9\% hombres y $53,1 \%$ mujeres).

La causa específica responsable de un mayor número de sentencias judiciales fue las alteraciones osteoarticulares postratamiento $(43,6 \%)$ que comprendían alteración de movilidad articular, dolor residual, etc., seguidas de la lesión vascular o nerviosa (34\%) y de las infecciones $(11,9 \%)$.

Solo un pequeño porcentaje $(2,3 \%)$ fue por un síndrome compartimental.

La lesión reclamada vía judicial ocurrió con más frecuencia en miembros inferiores $(56,5 \%)$ siendo la rodilla la zona más frecuentemente implicada.

La etiología de la lesión fue traumática en un 56,85\% de los casos y ortopédica (cirugía protésica, deformidades óseas no traumáticas como el hallux valgus, patología no traumática de columna...) en el 43,2\%.

En el escrito de reclamación la cuantía económica solicitada con más frecuencia como indemnización fue mayor de 100.000 euros en el $44,8 \%$ de los casos.

En el $94,7 \%$ de los casos la reclamación fue contra un especialista en traumatología de forma aislada sin combinación con otros médicos u otros profesionales. Además en el $78,2 \%$ de los casos solo se implicó a un único profesional.

Dentro de las causas generales el hecho imputado con más frecuencia en la reclamación judicial en segunda instancia fue el error terapéutico quirúrgico seguido del error diagnóstico.

En un nada desdeñable $14,9 \%$ el hecho motivo de la reclamación fue falta de consentimiento informado (40 casos) o consentimiento informado genérico ( 5 casos).

El daño reclamado fue en el $89,1 \%$ de los casos por lesiones, seguido de muerte en el 6,6\% y de daño moral en el $4,3 \%$.

En Traumatología y Cirugía Ortopédica en las sentencias emitidas en segunda instancia existió condena en el 49,8\% de los casos, siendo la cuantía de la indemnización mayoritariamente menor de 50.000 euros $(52,6 \%)$.

La cuantía media de la indemnización fue de 81.767 euros con un máximo de 867.000 euros y un mínimo de 600 euros.

Las dos mayores condenas fueron de 800.000 euros y 867.000 euros.

Llama la atención que en estas dos condenas el acto imputado fue una inexistencia de consentimiento informado con resultado de muerte del paciente.

En el primer caso la causa específica reclamada fue la lesión vascular en un procedimiento ortopédico de rodilla y en el segundo caso la lesión nerviosa en un procedimiento ortopédico de columna lumbar.

Se impuso el pago de costas judiciales en el $43,2 \%$ de los casos.

Respecto al contraste de variables en los cruces a través de las tablas de contingencia de Chi cuadrado destacan los siguientes resultados:

Al relacionar el tipo de jurisdicción con la existencia de condena destaca un menor número de condenas en la jurisdicción penal y un mayor número de condenas en la jurisdicción civil.

Cruzando el acto imputado con la jurisdicción destaca como resultados estadísticamente significativos que es la jurisdicción civil donde con más frecuencia se presentan demandas por defecto de información y en la jurisdicción contenciosa administrativa por error diagnóstico.

Al relacionar el acto imputado con la existencia de condena existe una clara relación entre defecto de información y condena.

En todos los 3 casos con diagnóstico de gangrena gaseosa se registró condena al traumatólogo.

Así mismo también destaca la no condena mayoritaria en los casos en que el acto imputado fue error terapéutico quirúrgico o error terapéutico ortopédico.

En el cruce entre el ámbito asistencial y la existencia de condena los datos no fueron estadísticamente significativos pero sí resultó significativo el cruce entre el ámbito asistencial con el acto imputado predominando el defecto de información y el error diagnóstico en el ámbito privado.

En el cruce entre el acto imputado y la cuantía de la condena se observan cuantías de condena menores $(<50.000$ euros) en el caso de error diagnóstico y cuantías moderadas (50.000-100.000) en el caso de error terapéutico quirúrgico.

En cuanto a la relación entre la causa específica y la existencia de condena, no se evidencian resultados estadísticamente significativos pero sí se evidencian en el cruce entre causa específica y cuantía de la condena existiendo con más frecuencia condenas $>$ de 100.000 euros en el caso se síndrome compartimental y condenas menores de 50.000 en alteraciones osteoarticulares residuales. 
Tabla 1 Variables administrativas

\begin{tabular}{|c|c|c|c|}
\hline Variable & Categorías & Número & Porcentaje \\
\hline \multicolumn{4}{|c|}{ Jurisdicción $(n=303)$} \\
\hline & Civil & 268 & 88,4 \\
\hline & Penal & 18 & 5,9 \\
\hline & Contencioso-Administrativa & 17 & 5,6 \\
\hline \multicolumn{4}{|c|}{ Fecha de la sentencia $(n=303)$} \\
\hline & 1995-1999 & 99 & 32,6 \\
\hline & $2000-2003$ & 101 & 33,3 \\
\hline & $2004-2011$ & 103 & 33,9 \\
\hline \multicolumn{4}{|c|}{ Comunidad Autónoma $(n=303)$} \\
\hline & Cataluña & 87 & 28,7 \\
\hline & Madrid & 35 & 11,6 \\
\hline & País Vasco & 25 & 8,3 \\
\hline & Andalucía & 35 & 11,6 \\
\hline & Otras & 121 & 39,9 \\
\hline \multicolumn{4}{|c|}{ Tribunal $(n=303)$} \\
\hline & Audiencia Provincial & 230 & 75,9 \\
\hline & Tribunal Superior de Justicia & 17 & 5,6 \\
\hline & Tribunal Supremo & 56 & 18,5 \\
\hline \multicolumn{4}{|c|}{ Ámbito asistencial $(n=303)$} \\
\hline & Público & 110 & 36,3 \\
\hline & Privado & 105 & 34,75 \\
\hline & Mutua & 61 & 20,1 \\
\hline & Sociedad médica & 27 & 8,9 \\
\hline
\end{tabular}

Cuando el resultado es la muerte las indemnizaciones menores de 50.000 euros son raras y sin embargo en el caso de daños morales las indemnizaciones mayoritariamente fueron menores de 50.000 euros.

\section{Discusión}

El estudio global de la mala praxis en traumatología y cirugía ortopédica en España es un trabajo complejo.

Tabla 2 Variables clínicas

\begin{tabular}{|c|c|c|c|}
\hline Variable & Categorías & Número & Porcentaje \\
\hline \multicolumn{4}{|c|}{ Sexo del paciente $(n=303)$} \\
\hline & Hombre & 142 & 46,9 \\
\hline & Mujer & 161 & 53,1 \\
\hline \multicolumn{4}{|c|}{ Causas específicas motivo de la reclamación. $(n=303)$} \\
\hline & Infección & 36 & 11,9 \\
\hline & Lesión vascular o nerviosa & 103 & 34 \\
\hline & Síndrome compartimental & 7 & 2,3 \\
\hline & Complicaciones sistémicas & 25 & 8,3 \\
\hline & Alteración osteoarticular & 132 & 43,6 \\
\hline \multicolumn{4}{|c|}{ Localización general de la sintomatología $(N=303)$} \\
\hline & Cadera & 36 & 11,9 \\
\hline & Rodilla & 59 & 19,5 \\
\hline & Pie-tobillo & 50 & 16,5 \\
\hline & Otros MMII & 26 & 8,6 \\
\hline & MMSS & 66 & 21,8 \\
\hline & Columna vertebral & 66 & 21,8 \\
\hline \multicolumn{4}{|c|}{ Patología $(n=303)$} \\
\hline & Ortopédica & 131 & 43,2 \\
\hline & Traumatológica & 172 & 56,8 \\
\hline
\end{tabular}


Tabla 3 Variables judiciales

\begin{tabular}{|c|c|c|c|}
\hline Variable & Categorías & Número & Porcentaje \\
\hline \multicolumn{4}{|c|}{$N .^{\circ}$ de profesionales implicados $(n=303)$} \\
\hline & Uno & 237 & 78,2 \\
\hline & Más de uno & 49 & 16,2 \\
\hline & Número no definido & 17 & 5,6 \\
\hline \multicolumn{4}{|c|}{ Tipo de profesional demandado $(n=303)$} \\
\hline & Traumatólogo & 287 & 94,7 \\
\hline & Traumatólogo + otro facultativo & 11 & 3,6 \\
\hline & Traumatólogo + otro estamento & 5 & 1,7 \\
\hline \multicolumn{4}{|c|}{ Causas generales motivo de la reclamación $(n=303)$} \\
\hline & Defecto de información & 45 & 14,9 \\
\hline & Error diagnóstico & 52 & 17,2 \\
\hline & Error terapéutico ortopédico & 41 & 13,5 \\
\hline & Error terapéutico quirúrgico & 114 & 37,6 \\
\hline & Mal seguimiento & 50 & 16,5 \\
\hline & Lado equivocado & 1 & 0,3 \\
\hline \multicolumn{4}{|c|}{ Resultado del daño reclamado $(n=303)$} \\
\hline & Muerte & 20 & 6,6 \\
\hline & Lesiones & 270 & 89,1 \\
\hline & Daños morales & 13 & 4,3 \\
\hline \multicolumn{4}{|c|}{ Cuantía económica de la demanda $(n=125)$} \\
\hline & $<50.000 €$ & 27 & 21,6 \\
\hline & $50.000-100.000 €$ & 42 & 33,6 \\
\hline & $>100.000 €$ & 56 & 44,8 \\
\hline \multicolumn{4}{|c|}{ Cuantía de la indemnización $(n=135)$} \\
\hline & $<50.000 €$ & 71 & 52,6 \\
\hline & $50.000-100.000 €$ & 38 & 28,1 \\
\hline & $>100.000 €$ & 26 & 19,3 \\
\hline \multicolumn{4}{|c|}{ Condena $(n=303)$} \\
\hline & Sí & 151 & 49,8 \\
\hline & No & 152 & 50,2 \\
\hline \multicolumn{4}{|c|}{ Imposición de costas $(N=303)$} \\
\hline & Sí & 192 & 43,2 \\
\hline & No & 111 & 56,8 \\
\hline
\end{tabular}

Para valorar el problema desde una perspectiva global precisaríamos conocer los datos de sentencias en primera instancia, sentencias en segunda instancia, reclamaciones extrajudiciales etc. pero no existe ningún tipo de archivo nacional donde estos datos estén reflejados, siendo deseable la existencia de bases de datos más completas ${ }^{2,12}$.

Disponemos de diferentes perspectivas para aproximarnos a este problema:

- Estudios de aseguradoras.

- Estudios de Colegios de médicos.

- Estudios de sentencias.

- Reclamaciones hospitalarias.

Aunque obviamente ninguna de ellas estudia el problema de forma global nos proporcionan datos muy útiles para usarlos en la práctica médica traumatológica diaria.

Fuera de la perspectiva puramente traumatológica también disponemos de estudios sobre efectos adversos y seguridad clínica, útiles para identificar posibles riesgos sanitarios muy relacionados con las futuras reclamaciones por negligencias médicas ${ }^{14}$ y estudios desde la perspectiva legal de los magistrados, más encaminados a definir la imprudencia médica y sus grados ${ }^{15}$.

La Traumatología y Cirugía Ortopédica es una especialidad de alto riesgo judicial.

En el estudio realizado sobre las quejas presentadas ante la Comisión Nacional de Arbitraje Médico ${ }^{16}$ procedentes de México, Distrito Federal, de un número total de 1.925 quejas se asignaron a la especialidad de Traumatología y Ortopedia 282 casos, lo que supone un $14,64 \%$ de las mismas y sitúa esta cifra en un porcentaje similar al de nuestra serie $(15,69 \%)^{2,17}$ situándose en primer lugar del total de reclamaciones por especialidades en España superando a la Ginecología-Obstetricia.

En este mismo sentido se pronuncia Álvarez González et al. ${ }^{18}$ el cual usa datos obtenidos del Instituto Médico Legal de Málaga correspondientes a denuncias por mala praxis en 
esta provincia y refleja que, por especialidades, en primer lugar destaca la Traumatología.

En Cataluña se han realizado estudios más centralizados dependientes del colegio de médicos de Cataluña, donde con datos de 24.000 médicos asegurados se recogieron en el periodo 1986-2005, 5.246 reclamaciones concluyendo ${ }^{12}$ que el $1,33 \%$ de los médicos asegurados se enfrentaron a alguna reclamación si bien solo el 0,26\% tuvo que abonar una indemnización al reclamante.

Este dato es muy diferente al número de condenas que refleja nuestra serie de sentencias en segunda instancia donde las condenas alcanzan el $49,8 \%$ de los casos ya que en nuestra serie se recogen los casos más complejos que han precisado el uso de la vía judicial y no solo de una primera instancia sino de una posterior revisión en segunda instancia.

Aun así ambas series reflejan unas cuantías indemnizatorias habituales menores de 50.000 euros $^{12}$ (en nuestra serie un $52,6 \%$ de condenas fueron menores de 50.000 euros).

En nuestro estudio el coste medio de las cuantías indemnizatorias es de 81.767 euros con un máximo de 867.000 euros y un mínimo 600 euros, al que habría que sumar el coste de las costas judiciales que se impusieron en el $43,2 \%$ de los procedimientos. En un reciente estudio publicado en el New England Journal of Medicine ${ }^{19}$ sobre el riesgo de mala praxis por especialidades, se sitúa la media de indemnizaciones considerando todas las especialidades reclamadas en 274.887 dólares (207.350 euros), cifra por lo tanto muy superior a la nuestra.

Las compañías aseguradoras de forma mayoritaria establecen un límite indemnizatorio de 600.000 euros por siniestro, estando por tanto en la mayoría de los casos el profesional cubierto por su seguro, pero es la Traumatología y Cirugía Ortopédica una de las pocas especialidades que sobrepasan este límite aunque únicamente en un $0,6 \%$ de los casos.

Las secuelas permanentes $(89,1 \%)$ son más reclamadas que el fallecimiento $(6,6 \%)$, porcentaje este claramente menor que el registrado en otras especialidades médicas o quirúrgicas (Neurocirugía, Psiquiatría.) probablemente en relación con el tipo de patologías diagnosticadas y tratadas en la traumatología cuyo riesgo vital (exceptuando los pacientes politraumatizados) es frecuentemente menor que en otras especialidades.

El hecho de que se produzca la muerte del paciente no inclina la balanza hacia la condena como cabría esperar y como ocurre en otras especialidades (porcentajes similares entre condena y no condena en traumatología) pero sí tiene un gran peso en la cuantificación de la indemnización que lógicamente son mayoritariamente más elevadas alcanzando en algunos casos los 800.000 euros.

La condena por daño moral $(4,3 \%)$ es poco relevante en traumatología.

En nuestra serie la mayoría de las demandas que prosperan tienen como objetivo la compensación económica presentándose el $88,4 \%$ de las demandas en el orden civil encontrándose solo en el orden penal un $5,9 \%$ de los casos. Este estudio recoge las sentencias en segunda o última instancia contra traumatólogos, lo que quiere decir que muchas reclamaciones vía penal o no se admiten a trámite y se archivan o no se condena en la primera instancia, puesto que el volumen de reclamaciones presentadas en el ámbito penal es muy superior.

En este sentido nuestros datos son superponibles a los obtenidos en el ámbito europeo, en Italia ${ }^{20}$, donde la mayor parte de las reclamaciones judiciales presentaban como único objetivo la indemnización.

- El ámbito privado es en el que con mayor frecuencia se producen las reclamaciones (más del $60 \%$ de los casos), probablemente debido al perfil del usuario de la sanidad privada que muchas veces relaciona pago de servicio con garantía de resultado.

- En cuanto a los motivos de la demanda judicial el error terapéutico quirúrgico es el más frecuente $(37,6 \%$ de los casos) seguido del error diagnóstico y el mal seguimiento del paciente.

Si valoramos conjuntamente el error terapéutico ortopédico y quirúrgico el porcentaje asciende al 51\% motivándose pues más de la mitad de las reclamaciones en errores en el tratamiento.

El defecto de información o comunicación es el motivo alegado en la reclamación judicial en el $14,9 \%$ de los casos y en el 77,8\% de ellos se acompañó de condena del traumatólogo. Este dato debe afianzar la corriente de la relación entre una práctica médica segura y la buena comunicación con el paciente.

En este sentido el consentimiento informado ${ }^{21}$; documento que hace que el paciente refleje por escrito que ha sido informado y que consiente en el procedimiento no evita las reclamaciones pero sí las agrava. No debemos centrarnos únicamente en la firma del consentimiento informando ya que un documento en el que se demuestre que no hubo comprensión por parte del paciente o que se realizó con premura o sin la debida anticipación puede ser invalidado ${ }^{8}$, por ello es fundamental reflejar en la historia clínica del paciente que se ha informado al mismo con comprensión por parte de este del procedimiento a realizar y sus posibles complicaciones. Una mala comunicación con el paciente incrementa enormemente el riesgo de litigio.

Un análisis de las demandas hechas en Estados Unidos demostró que un factor independiente que distinguía la existencia o no de demandas era el número de minutos dedicado por el médico a la entrevista, siendo la media de 15,0 en los demandados y de 18,3 en los no demandados. Factores involucrados en la forma de la relación entre el médico y el enfermo, como el tono de voz, también parecen ser determinantes.

- El síntoma o proceso específico que padecía el paciente que interpuso la reclamación judicial fue mayoritariamente la alteración osteoarticular tras el tratamiento $(43,6 \%)$ consistente en pérdida de movilidad articular, dolor residual etc., seguido de la lesión vasculonerviosa (34\%) y de la infección en el $11,9 \%$ de los casos.

Cuando la infección fue una gangrena gaseosa por Clostridium perfringens la condena ocurrió en el $100 \%$ de los casos frente al $58 \%$ en el total de las infecciones.

En el caso de lesión neurovascular la condena ocurrió en el $55 \%$ de los casos. 
En los síndromes compartimentales la condena ocurrió en el $57 \%$ de los casos registrándose indemnizaciones $>100.000$ euros en el $75 \%$ de los casos, probablemente en relación con las secuelas incapacitantes tras estos síndromes.

- El mayor número de reclamaciones interpuestas tiene como localización anatómica los miembros inferiores $(56,5 \%)$ siendo la rodilla la más frecuentemente implicada.

Con los resultados obtenidos en nuestro estudio aun tratándose de revisión de sentencias en segunda instancia podemos describir un perfil de situación especialmente proclive a las reclamaciones médicas en la traumatología y cirugía ortopédica y en la que los cirujanos ortopédicos en nuestro día a día deberíamos extremar las precauciones:

- Paciente usuario de la sanidad privada.

- Perteneciente a la comunidad autónoma catalana o madrileña.

- Se interviene quirúrgicamente de patología ortopédica o traumatológica.

- La localización es en la columna o miembros inferiores.

- El resultado posquirúrgico es insatisfactorio.

- El paciente no ha sido correctamente informado o no ha percibido una fluida comunicación y relación médicopaciente.

\section{Conclusiones}

- La Traumatología y Cirugía Ortopédica es una especialidad proclive a las reclamaciones por mala praxis tanto en su ámbito traumatológico como en el ortopédico.

- El número de condenas a traumatólogos es elevado pero las indemnizaciones suelen ser mayoritariamente menores de 50.000 euros, siendo cubiertas en la mayor parte de los casos por los seguros contratados por los profesionales médicos.

- El motivo fundamental de las reclamaciones es el error terapéutico quirúrgico siendo pues el acto quirúrgico fundamental y donde se deben extremar las precauciones.

- Las condenas por déficit de información o comunicación son elevadas por ello es importante fomentar una adecuada comunicación médico-paciente y rellenar correctamente el documento de consentimiento informado cerciorándose de que el paciente ha comprendido el procedimiento a realizar y sus posibles complicaciones siendo un gesto adecuado añadir en la historia clínica referencia a este extremo.

- El mayor número de reclamaciones tiene que ver con la satisfacción económica, especialmente en la práctica privada, predominando las reclamaciones en la jurisdicción civil, por ello es fundamental disponer de un seguro de responsabilidad civil con carácter retroactivo y que disponga de una cobertura prolongada en el tiempo.

- Los procesos más graves como gangrena gaseosa, síndrome compartimental, etc. donde la rapidez del diagnóstico y tratamiento devienen fundamentales son base de gran número de condenas a traumatólogos.

\section{Limitaciones del estudio}

Nuestro estudio tiene en cuenta únicamente las sentencias judiciales emitidas en segunda instancia sin entrar a considerar ni las sentencias emitidas en primera instancia ni las reclamaciones contra traumatólogos en las que se llega a un acuerdo extrajudicial.

Estos datos hubieran sido un complemento perfecto al presente estudio para valorar de forma global y completa las reclamaciones por mala praxis frente a traumatólogos y cirujanos ortopédicos pero no se han podido incluir dado que no existe ningún tipo de archivo nacional donde queden reflejados.

En este sentido autores españoles ${ }^{2,12}$ subrayan la necesidad de bases de datos así como estudios locales y nacionales.

\section{Responsabilidades éticas}

Protección de personas y animales. Los autores declaran que para esta investigación no se han realizado experimentos en seres humanos ni en animales.

Confidencialidad de los datos. Los autores declaran que han seguido los protocolos de su centro de trabajo sobre la publicación de datos de pacientes.

Derecho a la privacidad y consentimiento informado. Los autores declaran que en este artículo no aparecen datos de pacientes.

\section{Nivel de evidencia}

Nivel de evidencia III.

\section{Conflicto de intereses}

Los autores declaran no tener ningún conflicto de intereses.

\section{Bibliografía}

1. León-Sanz P. Ética médica y cirugía ortopédica y traumatología. Trauma Fund MAPFRE. 2010;21:65-74.

2. Perea Pérez B, Santiago Sáez A. El medico ante los tribunales: análisis de las sentencias judiciales relacionadas con la responsabilidad profesional medica en España. Rev Esp Med Legal. 2013;39:130-4.

3. Torre D. El seguro de responsabilidad civil. Actas del XII Encuentro de Responsabilidad Sanitaria. Madrid: Unidad Editorial; 2008.

4. Fenn P. Counting the cost of medical negligenc. BMJ. 2002;325:233-4.

5. Sethi MK, Obremskey WT, Natividad H, Mir HR, Jahangir AA. Incidence and costs of defensive medicine among orthopedic surgeons in the United States: A national survey study. Am J Orthop. 2012;41:69-73.

6. Hudson MJ, Moore GP. Defenses to malpractice: What every emergency physician should know. J Emerg Med. 2011;41:598-606.

7. Suk M, Udale AM, Helfet DL. Orthopaedics and the law. J Am Acad Orthop Surg. 2005;13:397-406.

8. Biberthaler P, Seifert J, Post M, Smektala R, Ottmann K, Braun $A$, et al. Identification of risk factors for subsequent legal claims in orthopedic and trauma surgery. Unfallchirurg. 2011;114:768-75. 
9. Gidwani S, Zaidi SM, Bircher MD. Medical negligence in orthopaedic surgery: a review of 130 consecutive medical negligence reports. J Bone Joint Surg Br. 2009;91:151-6.

10. Khan IH, Jamil W, Lynn SM, Khan OH, Markland K, Giddins G. Analysis of NHSLA claims in orthopedic surgery. Orthopedics. 2012;35:726-31.

11. Attarian DE, Vail TP. Medicolegal aspects of hip and knee arthroplasty. Clin Orthop Relat Res. 2005:72-6.

12. Arimany-Manso J, Gómez-Durán EL, Aubia Marimon J. Las reclamaciones sobre responsabilidad profesional por especialistas en un escenario diferente al estadounidense. Gac Sanit. 2013;27:92-3.

13. García-German Vazquez D, Sanz-Martín J. Cirugía en sitio erróneo. Rev Ortop Traumatol. 2009;53:332-9.

14. Grau J, Santiña M. Fundamentos de seguridad clínica en cirugía ortopédica y traumatología. Rev Ortop Traumatol. 2010;54:251-8.

15. Martín Pallín JA. Riesgo y responsabilidad en Cirugía ortopédica y Traumatologia. Rev Ortop Traumatol. 2003;53:210-23.

16. Valdés-Salgado R, Molina-Leza J, Solís-Torres C. Aprender de lo sucedido. Análisis de las quejas presentadas ante la
Comisión Nacional de Arbitraje Médico. Salud pública de México. 2001;43:444-54.

17. Perea Pérez B, Santiago Sáez A. Análisis de las consecuencias médico-legales de las reclamaciones judiciales de pacientes. Estudio comparativo de los casos de traumatología, ginecología y obstetricia, anestesia y reanimación y odontoestomatología. Trauma Fund MAPFRE. 2009;20: 264-8.

18. Alvarez Gonzalez F, Hidalgo Salvador E. Estudio retrospectivo de las denuncias por malpraxis médica en la provincia de Málaga. Cuad Med Forense. 2007;13:9-19.

19. Patel P, Robinson BS, Novicoff WM, Dunnington GL, Brenner MJ, Saleh KJ. The disruptive orthopaedic surgeon: Implications for patient safety and malpractice liability. J Bone Joint Surg Am. 2011;93:1261-6.

20. Umberto Tarantino MD, Alessio Gia Via MD. Professional liability in orthopaedics and traumatology in Italy. Clin Orthop Relat Res. 2013;471:3349-57.

21. Bhattacharyya T, Yeon H, Harris MB. The medical-legal aspects of informed consent in orthopaedic surgery. J Bone Joint Surg Am. 2005;87:2395-400. 